\title{
UV Degradation of Sesquiterpene Lactones in Chicory Extract: Kinetics and Identification of Reaction Products by HPLC-MS
}

\author{
Urban Frey ${ }^{\mathrm{a}}$, Johanna Claude ${ }^{\mathrm{a}, \mathrm{b}}$, Simon Crelier ${ }^{\mathrm{b}}$, and Marcel-Alexandre Juillerat ${ }^{\mathrm{b}}$
}

\begin{abstract}
Inulin, a valuable food ingredient, can be extracted from chicory roots (Cichorium intybus L.). However, the direct use of this material is limited, because of the presence of extremely bitter, co-extracted sesquiterpene lactones. Lactucin, a major component, has been degraded by UV irradiation, a process which showed neither temperature (293-313 K) nor initial concentration dependence. An overall half life time of ca. 45 min was determined. The degradation product was identified by HPLC-MS and ${ }^{1} \mathrm{H}$ NMR and was the result of the addition of a water molecule on the lactucin $\mathrm{C}(1)-\mathrm{C}(10)$ double bond, with the $\mathrm{OH}$ group located at the $\mathrm{C}(10)$ position.
\end{abstract}

Keywords: Degradation product · HPLC-MS · Kinetics · Sesquiterpene lactones · UV degradation

\section{Introduction}

Today's consumers prefer foods which taste fresh and natural, contain little or no additives, are nutritious and safe, and bring added health benefits. Inulin and oligofructose are widely used in functional foods throughout the world for their health-promoting and technological properties. They are already key ingredients in a large number of commercial products, the range of which keeps growing due to bustling research and development activity in the field [1]. Some non-food applications have also been proposed for oligosaccharides which including drug delivery, cosmetics and mouth washes [2].

Inulin, which is widely known for its bifidogenic, cholesterol-lowering, texturing or fat replacing properties, is found in chicory roots [1]. A major issue in the re-

\footnotetext{
${ }^{*}$ Correspondence: Dr. U. Frey

Tel.: +41276068657

Fax: +4127606 8615

E-Mail: urban.frey@hevs.ch

aUniversity of Applied Sciences Valais (HEVs)

Chemistry Department,

Rte du Rawyl 64

$\mathrm{CH}-1950$ Sion

bNestlé Research Center
}

$\mathrm{CH}-, 1000$ Lausanne 26, Switzerland covery of food-grade inulin on an industrial scale are the extremely bitter, co-extracted sesquiterpene lactones 1-6 shown in Fig. 1. Yet these compounds are photolabile [3], and the complete disappearance of the bitterness after a UV treatment has been demonstrated [4]. The structure of the degradation product, which should be elucidated for obvious food safety reasons, was however not known in detail. The degradation of lactucin was characterised in the present study.

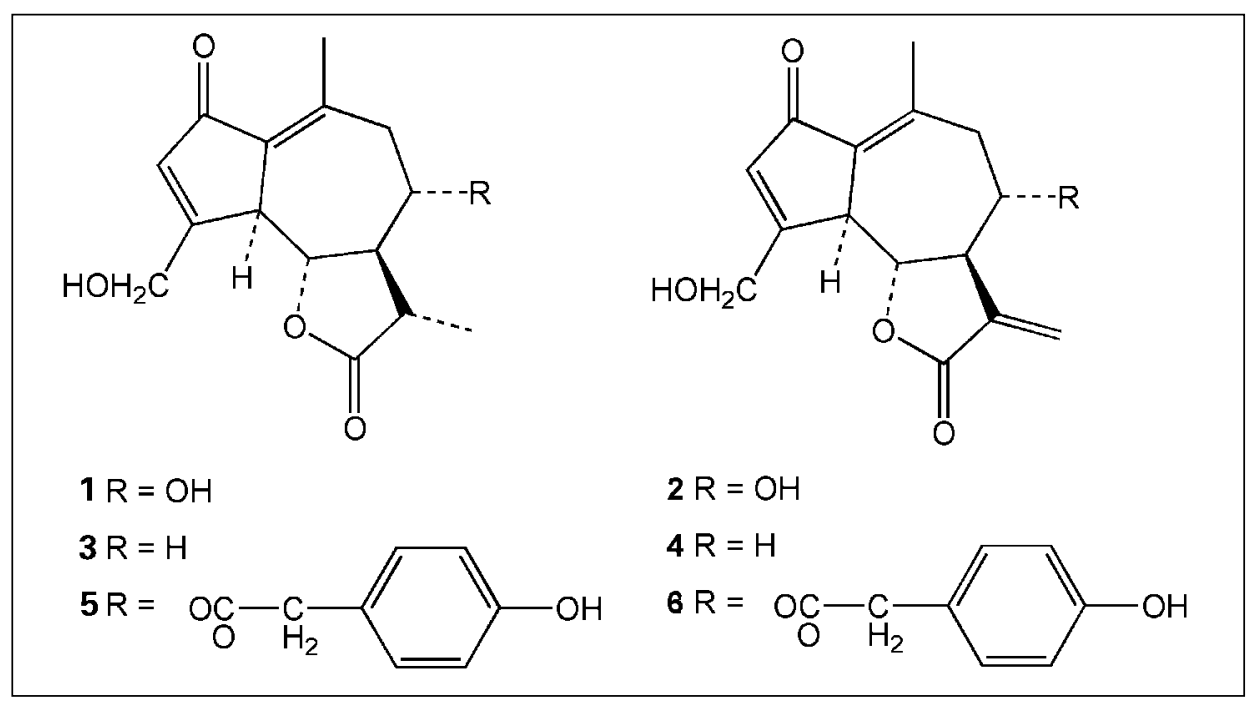

Fig. 1. Sesquiterpene lactones found in chicory root; 1: 11(S),13-dihydrolactucin; 2: lactucin; 3: 11(S),13-dihydro-8-deoxylactucin; 4: 8-deoxylactucin; 5: 11(S),13-dihydrolactucopicrin; 6: lactucopicrin

\section{Extraction and Purification of Lactucin}

Freeze-dried, powdered chicory roots (Cichorium intybus L.) were extracted with methanol. The methanol was removed and the extract dissolved in water, then treated with cellulase to release bound sesquiterpene lactones. The solution was successively extracted with dichloromethane and ethyl acetate to obtain a mixture of sesquiterpene lactones. This mixture was 
analysed by HPLC (UV detection at $262 \mathrm{~nm}$ ) coupled with mass spectrometry, i.e. MS analysis after atmospheric pressure chemical ionisation in the positive mode (APCI, pos). The mixture was further fractionated and for the present study, a solution of pure lactucin was obtained by semi-preparative HPLC.

\section{Kinetics of UV Degradation}

UV degradation experiments were carried out to determine the rate constant of the degradation and its dependence on temperature and lactucin concentration. Lactucin solutions were placed in quartz cuvettes and thermostated in a water bath. The absorbance spectra of lactucin (2) were then measured as a function of UV $(366 \mathrm{~nm})$ irradiation time. A decrease of the maximum at $260 \mathrm{~nm}$, an increase at $220 \mathrm{~nm}$ and an isosbestic point at $245 \mathrm{~nm}$ were observed (Fig 2). The presence of an isosbestic point indicates that only one (major) degradation product is obtained. The photodegradation of lactucin (2) follows a pseudo-first-order kinetics.

No temperature dependence of the rate constant between 293 and $313 \mathrm{~K}$ was observed. Also no rate dependence with respect to lactucin concentration (0.4-1.7 $\times$ $10^{-5} \mathrm{~g} / \mathrm{ml}$ ) was found. An overall rate constant of $2.6 \pm 0.4 \times 10^{-4} \mathrm{~s}^{-1}$ was determined, which corresponds to a half life of $c a$. $45 \mathrm{~min}$.

\section{Identification of Degradation Product}

HPLC analysis of a lactucin solution after the UV treatment showed that the sesquiterpene lactone degraded to one major compound, 7. The MS (APCI, pos) spectrum of this compound shows a molecular ion at m/z $295\left(\mathrm{M}+\mathrm{H}^{+}\right)$as well as the fragments already observed for lactucin (Fig 3). This corresponds to the addition of one water molecule to a double bond of lactucin, in accordance with the observed shift from 260 to $220 \mathrm{~nm}$ of the absorption wavelength maximum (Fig. 2). Analysing the ${ }^{1} \mathrm{H}$ NMR spectrum of the degradation product and comparing it to the spectrum of lactucin helped identify the double bond where the water addition takes place. The main difference between these two spectra is observed for the $\mathrm{C}(15)$ methyl group signal which is a singlet at $2.4 \mathrm{ppm}$ for lactucin (2). In the spectrum of 7, this signal is absent and two new singlets at $1.42 \mathrm{ppm}$ and $1.23 \mathrm{ppm}$ in a ratio of about 3:1 are observed. This change can be explained by the

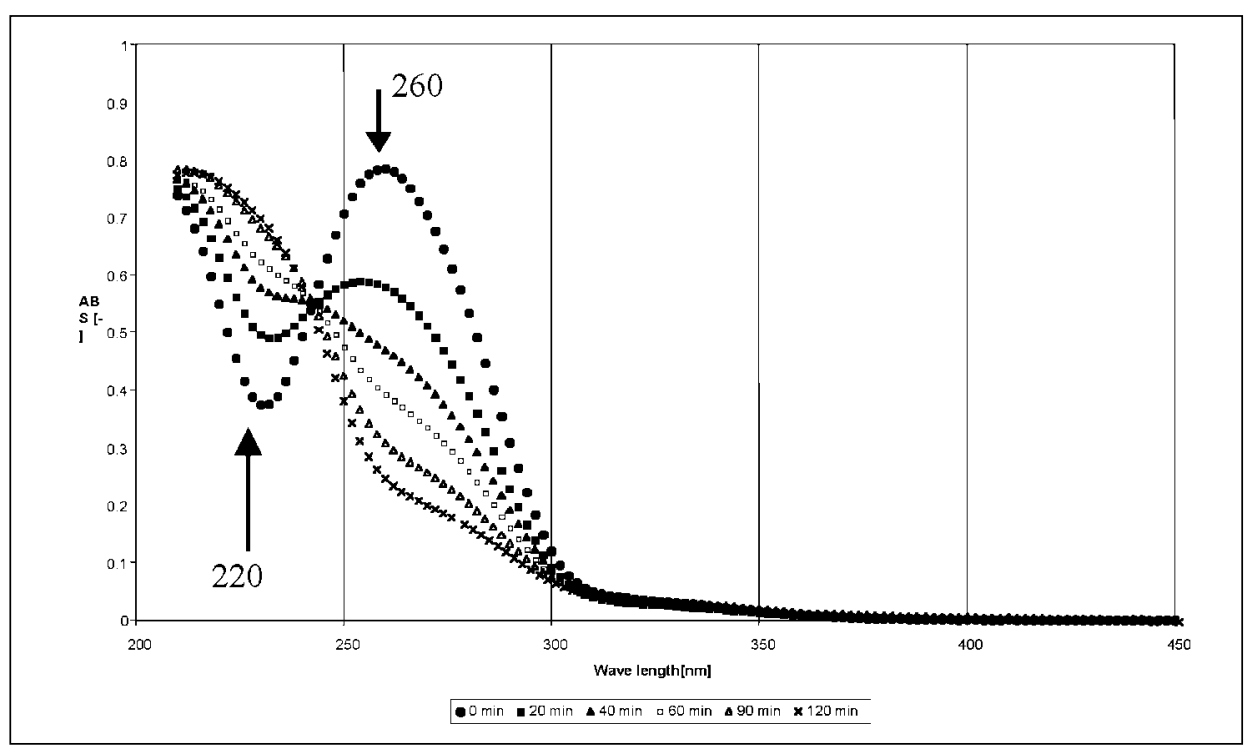

Fig. 2. UV-VIS spectra of lactucin (2) $\left(1.710^{-5} \mathrm{~g} / \mathrm{ml}\right)$ as a function of UV irradiation time at $303 \mathrm{~K}$.

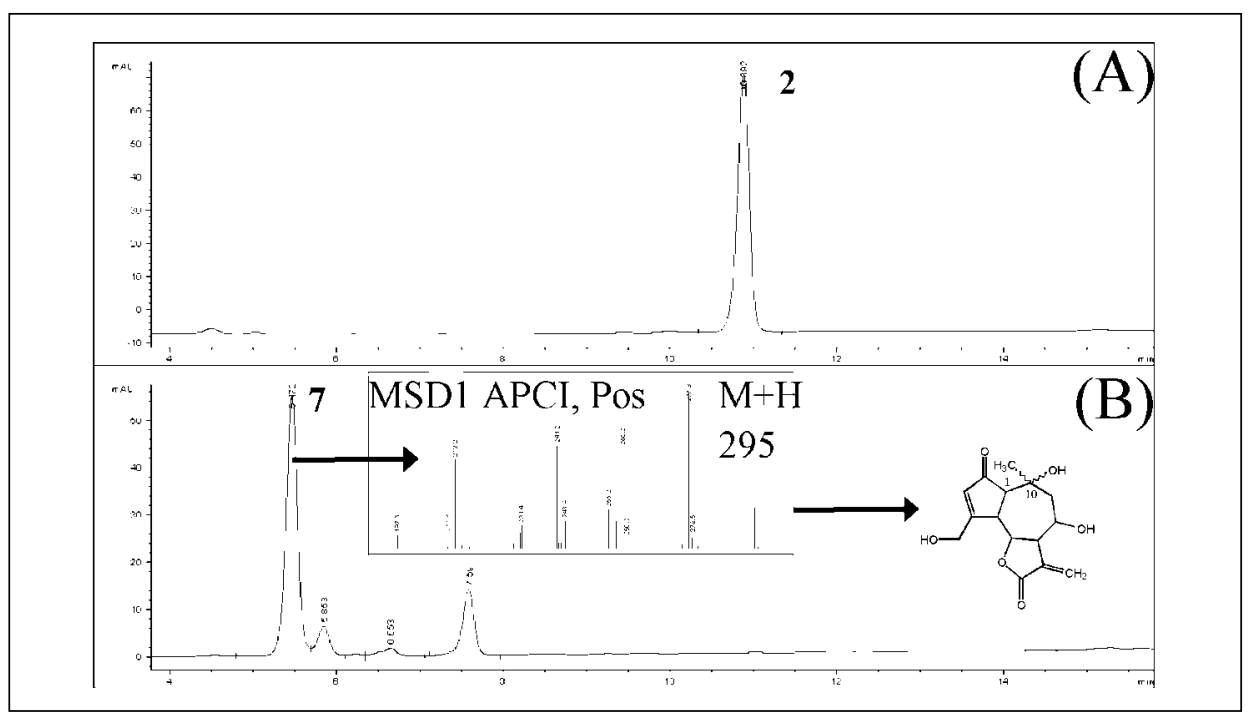

Fig. 3. Chromatograms of lactucin solution $\left(1.710^{-5} \mathrm{~g} / \mathrm{ml}\right)$ : (A) before and (B) after UV degradation. The insert shows the MS spectrum of the major degradation product and the proposed structure.

addition of one water molecule to the $\mathrm{C}(1)-\mathrm{C}(10)$ double bond, with the $\mathrm{OH}$ group located on $\mathrm{C}(10)$. The presence of two singlets can be explained by the two possible diastereomers, which result from the addition of one water molecule.

\section{Conclusion}

The bitter sesquiterpene lactones co-extracted with inulin from chicory roots can be completely degraded by UV treatment. The kinetics of the degradation process was measured in the case of lactucin (2). The unique degradation product has been identified using an HPLC-MS technique, com- pleted by analysis of ${ }^{1} \mathrm{H}-\mathrm{NMR}$ spectra. The degradation of lactucopicrin (6) and 8-deoxylactucin (4) very likely follows similar pathways. The food safety of these water adducts should be ascertained in view of future food applications.

Received: May 2, 2002

[1] K.R. Niness, J. Nutrition 1999, 7, 1402s.

[2] R.G. Crittenen, M.J. Playne, J. Food Science \& Technology 1996, 7, 353.

[3] E. Leclercq, A.G.J. Voragen, L.M. Brugging, W. Pilnik, Z. Lebensm. Unters. Forsch. 1988, 187, 440.

[4] P. Wilgotson, S. Crelier, Nestlé, personal communication, 1999. 